\title{
Mitochondrial autotransplantation: A "shot" in the dark?
}

\author{
James M. Hammel, MD
}

From the Department of Cardiothoracic Surgery, Children's Hospital and Medical Center, Omaha, Neb. Disclosures: Author has nothing to disclose with regard to commercial support.

Received for publication March 13, 2017; accepted for publication March 17, 2017; available ahead of print April $18,2017$.

Address for reprints: James M. Hammel, MD, Cardiothoracic Surgery, Children's Hospital and Medical Center, 8200 Dodge, Omaha, NE 68114 (E-mail: jhammel@chsomaha.org).

J Thorac Cardiovasc Surg 2017; 154:290

$0022-5223 / \$ 36.00$

Copyright $\subset 2017$ by The American Association for Thoracic Surgery

http://dx.doi.org/10.1016/j.jtcvs.2017.03.061

Considerable effort has been expended in the effort to understand and alter the outcomes of myocardial injury. $\mathrm{Ne}$ crosis itself is easy enough to grasp. But decades of research into the mechanisms of ischemia-reperfusion injury and apoptosis have revealed that the outcomes of sublethal stress of the cardiomyocyte are highly heterogenous and possibly amenable to therapeutic intervention. In their report in this issue of the Journal, Emani and colleagues ${ }^{1}$ describe their initial experience with autotransplantation of purified mitochondria in an effort to improve recovery of dysfunctional myocardium in desperately ill infants who did not have improvement during extracorporeal circulatory support. Did it work?

The body of experimental data supporting such an approach is substantial. Clearly, mitochondria change in structure and function after sublethal ischemia. Mitochondria can be harvested and purified, and uptake of transplanted mitochondria into viable cardiomyocytes can be demonstrated. Improved cellular viability and function can be shown in vitro and in carefully controlled animal studies. But several questions might trouble the "mitoskeptic":

- Mitochondria were harvested from a small mass of skeletal muscle, which is less rich in mitochondria to begin with than cardiac muscle. Was the number transplanted large enough to be significant?

- The process of skeletal muscle excision and mitochondrial isolation must involve ischemia and stress on the mitochondria. Why is this not just as damaging to the function of the mitochondria to be transplanted as the original injury was to the mitochondria of the heart?

- The process of injection of small volumes into multiple locations in the myocardium must obviously result in

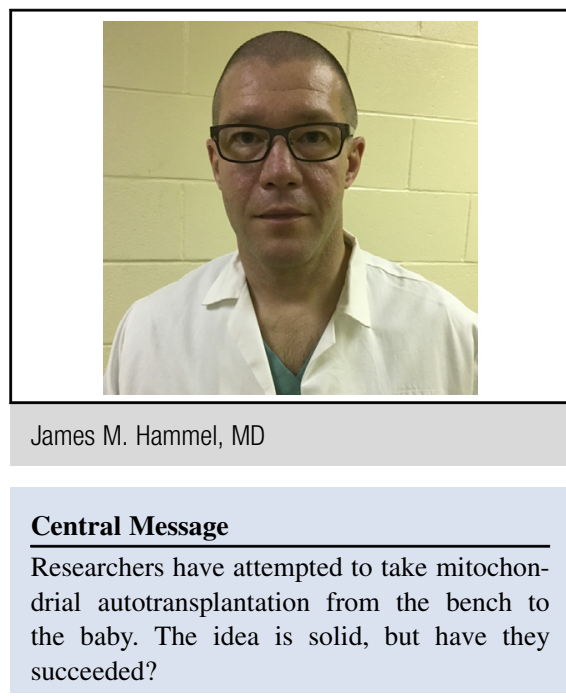

See Article page 286.

uneven distribution. What is a cardiomyocyte to do with too many or too few mitochondria?

- In our current understanding of apoptotic effector pathways, the mitochondrion is as much the target of cellular signaling after ischemia as it is a mediator. What prevents the transplanted mitochondria from being deactivated in the post-sublethal injury milieu?

The clinical experience is that with circulatory support, dysfunctional infant myocardium usually, but not always, improves after a sublethal injury. In this brief research report, Emani and colleagues ${ }^{1}$ bring to our attention the possibility that the fate of that injured muscle might be altered through the groundbreaking intervention of mitochondrial autotransplantation, that the procedure can be carried out without obvious ill effects, and that it might even work. Demonstrating efficacy will of course require a randomized, placebo-controlled, prospective trial.

\section{Reference}

1. Emani SM, Piekarski BL, Harrild D, del Nido PJ, McCully JD. Autologous mitochondrial transplantation for dysfunction after ischemia-reperfusion injury. $J$ Thorac Cardiovasc Surg. 2017;154:286-9. 